\title{
DIFFERENCES MORPHO-BIOCHIMIQUES OBSERVÉES SUR DEUX LOTS DE SOUCHES SAUVAGES DE CRYPTOCOCCUS ALBIDUS : V. ALBIDUS ET V. DIFFLUENS
}

\author{
H. SAËZ et T. L. NGUYEN
}

\begin{abstract}
RÉSUMÉ. Une investigation a été menée sur 115 souches sauvages de Cryptococcus albidus, comprenant 61 isolements de la variété albidus et 54 de la variété diffluens. L'étude morphologique est basée sur l'observation : de l'aspect muqueux des colonies, de la pigmentation, du développement en milieu liquide, de la forme des blastospores et de la filamentation. Quant aux caractères physiologiques, il a été rapporté les résultats concernant : la fermentation, l'assimilation de 24 produits hydro-carbonés et du nitrate de potassium, le développement en fonction : de la température, de deux taux d'actidione (cyclohexamide) et de cinq taux de tétrazolium. La discussion a porté sur l'atypicité et les variations intra-spécifiques, la distinction des deux variétés et la valeur taxonomique des deux entités.
\end{abstract}

\section{Morpho-biochemical differences observed on two wild strains groups of Cryp- tococeus albidus: V. albidus and V. diffluens.}

SUMMARY. The morpho-biochemical characteristics were studied on 115 wild strains of Cryptococcus albidus: 61 isolates of the variety albidus and 54 of the variety diffluens. The mucous aspect of the colony, the pigmentation, the development on liquid medium, the cells'form and the filamentation were the morphological characters observed. As for the physiology, were reported the results concerning: the fermentation, the assimilation of 24 carbohydrates and the potassium nitrate, the development according: the temperature, two rates of actidione (cycloheximide) and four rates of tetrazolium. Were discussed: the atypicity and the intra-specific variations, the differenciation of the varieties and the taxonomic value of the two entities.

Les Cryptococcus sont des levures asporogènes dont les frontières avec les Torulopsis et les Rhodotorula furent l'objet de nombreuses discussions. L'introduction d'un critère physiologique, l'assimilation de l'inositol, dans la définition générique, est, tout au moins sur le plan pratique, une initiative heureuse de Phaff et Spencer (in 5). Encore faut-il savoir que l'élection de ce produit par certaines souches peut être faible ou tardive.

Le $C$. diffluens, considéré encore comme une espèce par Lodder et Kreger-Van Rij (4), a été rétrogradé au rang de variété par Phaff et Fell (5). Parmi les trois variétés

Muséum national d'Histoire naturelle, Ethologie et Conservation des espèces animales, Parc Zoologique; 53, Avenue de Saint-Maurice F 75012 Paris

Accepté le 8 mai I98I. 
de $C$. albidus retenues par ces Auteurs, à savoir v. albidus, v. aerius et v. diffluens, seules d'eux d'entre elles vont retenir notre attention ; jusqu'ici, en effet, nous n'avons jamais rencontré aerius dans nos prélèvements.

L'étude comparée des variétés albidus et diffluens repose sur les résultats de deux lots, respectivement de 61 et 54 souches sauvages, toutes isolées au laboratoire et examinées aussitôt après purification des colonies apparues en primo-culture, sans passage pendant un certain temps sur milieux de conservation.

Pour ces 115 levures nous rapportons, successivement, nos observations sur : la morphologie, la fermentation, l'assimilation de 24 produits hydro-carbonés et du nitrate de potassium, la température maximale de développement, la croissance sur milieu à l'actidione (cycloheximide) (2 doses testées) et sur milieu au triphényltétrazolium (4 doses croissantes).

\section{Matériel et méthode}

L'échantillon albidus renferme : 32 isolements de mammifères et 21 d'oiseaux sauvages captifs, 1 de l'homme et 7 de l'atmosphère en trois endroits du Parc zoologique de Paris ; quant à celui de diffluens : 27 de mammifères, 16 d'oiseaux et 11 de l'atmosphère du Parc.

A propos de la différenciation de $C$. neoformans d'avec deux autres Cryptococcus voisins (7) nous avons décrit notre méthode de travail. Aussi rappelons-nous seulement ici que :

- les souches sont conservées 30 jours, à l'obscurité, à la température d'incubation de $25^{\circ}$;

- sauf indication d'un autre milieu, les résultats morphologiques sont ceux obtenus : sur la gélose de Sabouraud à $2 \%$ de glucose ou en eau peptonée à $1 \%$ et glucosée à $2 \%$;

- la fermentation, selon la technique de Guerra (bouchon de paraffine à la surface du milieu liquide), est recherchée avec les 9 produits : glucose, galactose, saccharose, maltose, cellobiose, tréhalose, lactose, raffinose et inuline ;

- l'assimilation des produits hydro-carbonés et du nitrate de potassium est étudiée en tubes, sur milieu gélosé-vitaminé ;

- la température maximale : en tubes, sur la gélose de Sabouraud; résultats après 10 jours d'incubation ;

- l'action de l'actidione à $0,50 \mathrm{~g}$ et $\mathrm{Ig} /$ litre : sur la gélose de Sabouraud, en tubes, en présence d'un tube témoin sans actidione; résultats après 10 jours à $25^{\circ}$;

- l'action du tétrazolium à $1 / 10000,2 / 10000,5 / 10000$ et $1 / 1000$ : sur la gélose de Sabouraud, en tubes, en présence d'un tube témoin sans tétrazolium.

Afin de mieux faire ressortir les différences ou les similitudes de ces deux variétés de $C$. albidus les résultats seront souvent indiqués en pourcentages. 


\section{Résultats}

\section{1 - CARActéristiques morphologiques. (tableau I)}

1 - Le caractère muqueux. Il est des souches dont la culture coule plus ou moins rapidement au fond du tube ; d'autres à colonie mucoïde mais non coulante ; d'autres enfin dépourvues de ce caractère. Cet aspect macroscopique s'observe en milieu solide et, quelquefois également, en milieu liquide quand le Cryptococcus se développe en surface et forme des îlots ou un voile épais. Le caractère muqueux varie non seulement avec la souche, mais encore avec le milieu de culture et la température d'incubation.

2 - Le développement à la surface du milieu liquide. Pour la variété albidus on note dans environ $50 \%$ des cas : soit l'absence de développement ou la présence d'un anneau parfois incomplet, soit la production d'îlots ou de voile quelquefois épais. Ilots et voile sont un peu moins fréquents dans la variété diffluens $(40,8 \%$ des souches contre $49,2 \%$ ).

3 - La filamentation. L'absence de filamentation est de règle pour les deux variétés ; la formation de pseudo-hyphes, exceptionnelle, n'a été vue que chez albidus. Entre ces cas limites il arrive de découvrir à l'examen microscopique, tantôt des tubes mycéliens rarement ramifiés, tantôt des ébauches de pseudo-filamentation.

4 - La forme des cellules. Les blastospores varient de la forme ronde à ovale en passant par les formes intermédiaires suivantes : sub-sphérique, ovoïde, courte ovale, longue ovale, plus rarement cylindrique. Dans la majorité des souches des deux variétés on trouve, en proportions différentes, des cellules rondes et ovales; cependant, c'est dans la variété diffluens que la forme ronde prédomine.

TABLEAU I. - Caractéristiques morphologiques (Pourcentages).

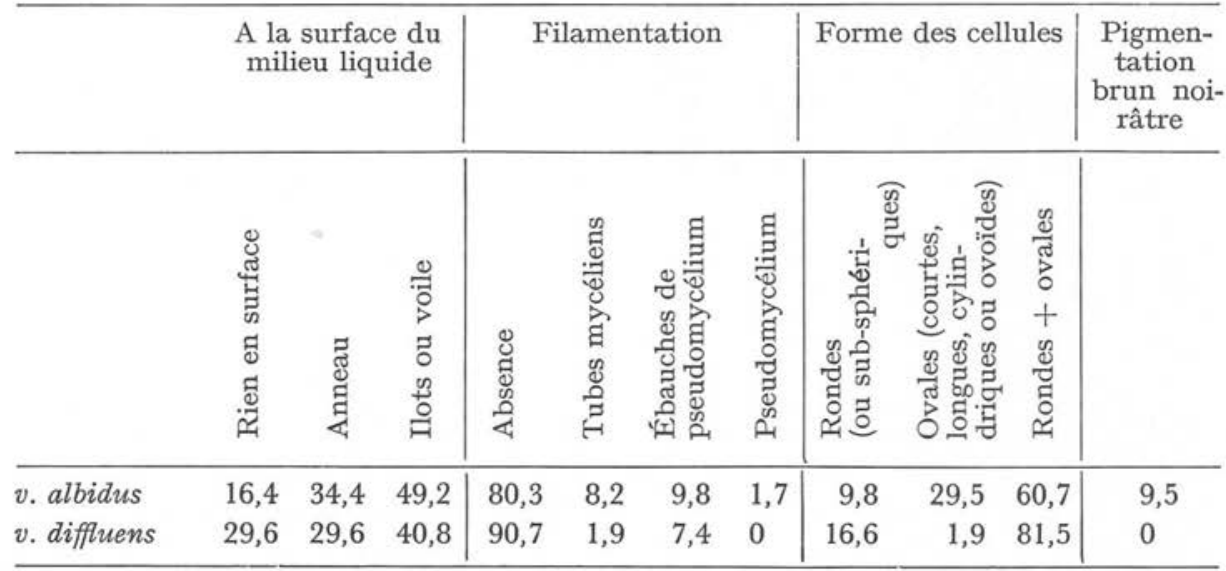


5 - La pigmentation inhabituelle. Une pigmentation brun noirâtre, tendant à diffuser dans la gélose, a été remarquée uniquement dans la variété albidus, dans un petit nombre de cas $(9,5 \%)$. Elle apparaît, généralement tardivement, sur les milieux de : Sabouraud, Gorodkowa, acétate de sodium ; elle n'a jamais encore été constatée sur une gélose au malt.

\section{2 - Fermentation.}

Absente chez les 115 souches testées.

3 - Assimilation des produits hydro-Carbonés (Voir tableau II).

D'après leur mode d'utilisation on distingue trois groupes de produits :

1 - Les produits ne permettant aucune distinction entre les deux variétés :

— assimilation par $100 \%$ des souches : 10 produits, à savoir : glucose, saccharose, maltose, cellobiose, tréhalose, mélézitose, D xylose, L arabinose, D sorbitol et inositol ;

— non assimilation par $100 \%$ des souches : l'inuline.

Il faut néanmoins souligner que l'utilisation peut n'être que faible et (ou) tardive parmi ces 10 produits assimilés. Par conséquent, même au niveau des réactions globalement communes, les deux variétés se particularisent ainsi :

- v. albidus, réaction + faible et (ou) tardive ; produits rangés selon l'ordre décroissant des pourcentages :

$\begin{array}{lrc}\text { tréhalose } & 14,7 \% \text { des souches } \\ \text { mélézitose } & 11,5 \% & - \\ \text { cellobiose } & 9,8 \% & - \\ \text { L arabinose } & 6,6 \% & - \\ \text { D sorbitol } & 5,0 \% & - \\ \text { inositol } & 1,6 \% & -\end{array}$

- v. diffluens, réactions + faible et (ou) tardive :

$\begin{array}{lrr}\text { tréhalose } & 50,0 \% \text { des sou } \\ \text { mélézitose } & 37,1 \% & - \\ \text { D sorbitol } & 24,1 \% & - \\ \text { cellobiose } & 14,8 \% & - \\ \text { inositol } & 5,4 \% & - \\ \text { maltose } & 3,6 \% & - \\ \text { saccharose } & 1,8 \% & - \\ \text { L arabinose } & 1,8 \% & - \\ \text { D xylose } & 1,8 \% & -\end{array}$

2 - Les produits permettant une distinction relative entre les deux variétés : - groupe de 12 produits pour lesquels la réaction est + ou $+/$ - dans l'une et l'autre variété, à savoir : érythritol, raffinose, mélibiose, alcool, alpha méthyl-D-glucoside, D mannitol, adonitol, galactose, L rhamnose, dulcitol, ribose et sorbose. 
TABLEAU II. - Caractèves communs et distinctifs de C. albidus : v. albidus et $\mathrm{v}$. diffluens, rangés d'après l'écart croissant de pourcentage obtenu entre les deux échantillons étudiés.

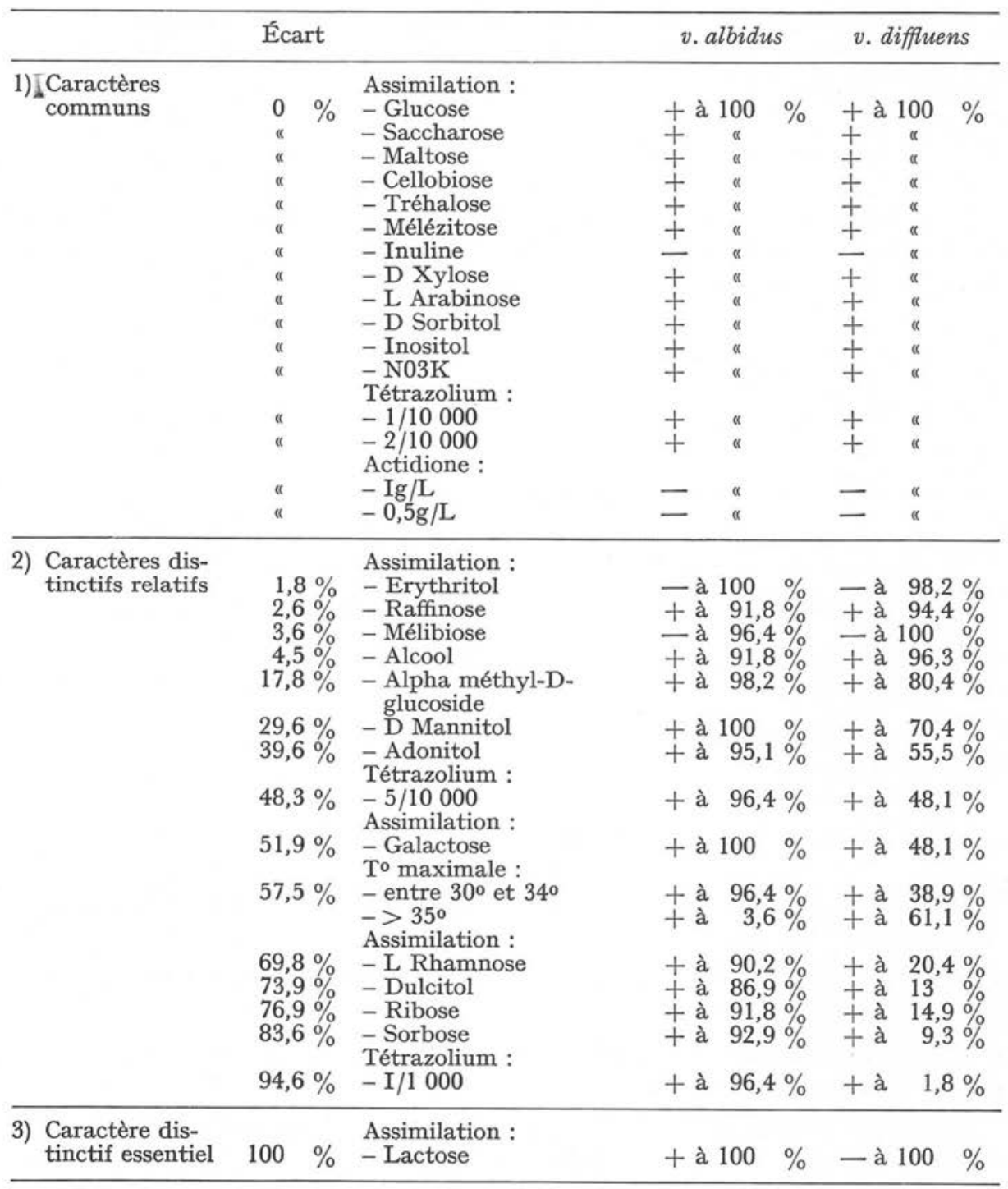

La distinction est d'autant plus significative que l'écart de pourcentage entre les deux échantillons est d'autant plus large. Ainsi l'écart, $1.8 \%$, est faible avec l'érythritol : $100 \%$ de réactions négatives avec la $v$. albidus et $92,2 \%$ avec la $v$. dif- 
fluens; l'écart majeur, 83,6\%, s'observe avec le sorbose assimilé à 92,9\% par la $v$. albidus et seulement à $9,3 \%$ par la $v$. diffluens.

3 - Le produit permettant la distinction entre les deux variétés :

- le lactose, qui n'est assimilé que par la $v$. albidus, mais $11,4 \%$ de ceux-ci n'ont donné qu'une réaction faible et (ou) tardive.

\section{4 - Assimilation du nitrate de potassium}

Le nitrate de potassium est, fortement et précocement, assimilé par la totalité des souches de la $v$. diffluens et par la majorité de celles de la $v$. albidus dont l'utilisation a été faible et (ou) tardive dans $4,9 \%$ des cas.

\section{5 - Temperature maximale}

Les températures maximales de développement varient à l'intérieur de l'intervalle suivant :

— v. albidus: $30^{\circ}-370: 96,4 \%$ poussent entre $30^{\circ}-34^{\circ}$

$\begin{array}{rrr}3,6 \% & - & 35^{\circ}-37^{\circ} \\ -v . \text { diffuens : } 32^{\circ}-38^{\circ}: 38,9 \% & - & 32^{\circ}-34^{\circ} \\ 61,1 \% & - & 35^{\circ}-38^{\circ}\end{array}$

\section{6 - DÉveloppement SUR Milieu ACTIDione}

Aucune des 115 souches étudiées ne s'est développée sur milieu contenant $0,5 \mathrm{~g} /$ litre et Ig/litre d'actidione.

\section{7 - DÉVELOPPEMENT SUR MILIEU TÉTRAZOLIUM}

Après 5 jours d'incubation à $25^{\circ}$ les souches des deux échantillons ont eu le comportement ci-après :

- sur milieu contenant $1 / 10000$ et 2/10 000 de tétrazolium : toutes les souches se développent ;

- sur milieu contenant 5/10 000 de tétrazolium : v. albidus se développe dans $96,4 \%$ des cas et $v$. diffluens dans $48,1 \%$;

- sur milieu contenant $1 / 1000$ de tétrazolium : $v$. albidus se développe encore à $96,4 \%$, tandis que $v$. diffluens ne le fait plus qu'à $1,8 \%$.

\section{Discussion}

Nos résultats concordent, dans l'ensemble, avec ceux de Phaff et Fell (5), à ceci près, par exemple, que le raffinose et l'alcool, rapportés comme toujours utilisés, ne 
le sont pas par quelques-unes de nos souches; nous revenons sur cette question à propos de l'atypicité. C'est sur la valeur accordée aux critères distinctifs relatifs (assimilation du : galactose, méthyl-D-glucoside et mélézitose) que nos points de vue divergent quelque peu. Nous allons discuter successivement de :

1 - l'atypicité des souches ;

2 - la distinction des deux variétés;

3 - du bien fondé de la séparation taxonomique des deux entités.

1 - Plusieurs publications ont été consacrées, ces dernières années, à des levures " atypiques ». Quand on isole un grand nombre de microorganismes d'habitats naturels divers il faut s'attendre à en trouver certains qui, par un caractère morphologique ou biologique, ne cadrent pas entièrement avec la description classique de l'espèce donnée. Entre les variations intra-spécifiques, l'atypicité et la mutation qui s'accompagne, parfois, d'un bouleversement de l'équipement enzymatique, il existe divers degrés de différences d'ordre quantitatif et qualitatif. Nous considérons plutôt comme des variations, parfois limites, mais intra-spécifiques, les résultats cidessous obtenus chez $1 \%$ à $10 \%$ de nos souches sauvages :

a - variations de l'ordre de $10 \%$ des souches :

- formation de tubes mycéliens ou de pseudomycélium rudimentaire : dans les 2 variétés ;

- production de pigment brun noirâtre : dans la v. albidus ;

b - variations de l'ordre de 1 à $5 \%$ des souches :

- formation de pseudomycélium : dans la v. albidus;

- assimilation du mélibiose : dans la v. albidus;

- assimilation de l'érythritol : dans la $v$. diffluens;

- non-assimilation du raffinose : dans les 2 variétés;

- non assimilation de l'alcool : dans les 2 variétés ;

- croissance sur milieu au tétrazolium à $1 / 1000$ : dans la v. diffluens;

— croissance au-dessus de $35^{\circ}$ : dans la v. albidus.

En 1965 nous signalions la possibilité de filamentation chez certains Cryptococcus (6). S'agissant de l'utilisation du mélibiose, nos résultats sont proches de ceux de Widra et Long, pour lesquels la réaction est négative avec les deux variétés. Dabbagh et coll. $(1,2)$, considèrent comme une croissance résiduelle celle observée à 370 en particulier chez $C$. diffluens; parmi les Cryptococcus dits saprophytes nous avons observé $(6,7)$, des signes de croissance à $37^{\circ}, 38^{\circ}$, voire, tout récemment, à $39^{\circ}$ chez un C. laurentii v. laurentii; Uden et Do-Carmo-Sousa (8) ont isolé du caecum de bovidés un $C$. diffluens se développant à $37^{\circ}$.

2 - Avant d'aborder la discussion des caractères distinctifs proprement dits, il nous faut attirer l'attention sur les résultats concernant l'inositol et le nitrate de potassium :

- l'assimilation de l'inositol est un critère générique des Cryptococcus; or, chez $1,6 \%$ des souches de la $v$. albidus et $5,4 \%$ de celles de la $v$. diffluens nous n'avons 
obtenu qu'une réaction faible et (ou) tardive ; une telle assimilation pourrait passer inaperçue au cours d'une diagnose rapide ;

- l'assimilation du nitrate de potassium est un critère spécifique de $C$. albidus; chez $4,9 \%$ des souches de la $v$. albidus la réaction a été faible et (ou) tardive.

De même que Kreger-Van Rij (3) nous pensons qu'il est hasardeux d'asseoir une distinction sur les seuls caractères morphologiques. Notons cependant que :

- le pigment brun noirâtre n'a été rencontré jusqu'ici que dans la v. albidus ;

- la forme ronde des blastospores se voit plus fréquemment dans la v. diffluens.

Quant aux tests physiologiques, les écarts de pourcentages supérieurs à $50 \%$ vont en augmentant ainsi :

- assimilation du galactose :

- croissance au-delà de $35^{\circ}$ :

- assimilation du L rhamnose :

- assimilation du dulcitol :

- assimilation du ribose :

- assimilation du sorbose :

- croissance sur tétrazolium à $1 / 1000$ :

- assimilation du lactose :

$51,9 \%$
$57,5 \%$
$69,8 \%$
$73,9 \%$
$76,9 \%$
$83,6 \%$
$94,6 \%$
$100,0 \%$

En se référant aux chiffres ci-dessus et aux tableaux II et III on s'aperçoit que : - l'élection du lactose constitue le seul critère vraiment distinctif : $100 \%$ d'assimilation par la $v$. albidus, $100 \%$ de non-assimilation par la $v$. diffluens ;

- néanmoins, le critère tétrazolium a une valeur voisine de celle du lactose : un C. albidus ne se développant pas sur milieu à $1 / 1000$ de tétrazolium a 98,2 chances sur 100 d'appartenir à la $v$. diffluens ; nous citerons encore le sorbose, pour ses probabilités encore fortes : ce produit est assimilé par $92,9 \%$ de nos souches de la $v$. albidus et non assimilé par $90,7 \%$ de celles de la $v$. diffuens.

TABLEAU III. - Caractéristiques morpho-physiologiques comparées de $\mathrm{C}$. albidus : $\mathrm{v}$. albidus $e t \mathrm{v}$. diffluens

\begin{tabular}{|c|c|c|}
\hline & v. albidus & v. diffluens \\
\hline $\begin{array}{l}1 \text { En milieu liquide : } \\
\text { - anneau } \\
\text { - ̂llots } \\
\text { - voile }\end{array}$ & $\begin{array}{l}+1- \\
+1- \\
+1-\end{array}$ & $\begin{array}{l}+1- \\
+1- \\
+1-\end{array}$ \\
\hline $\begin{array}{l}2 \text { En milieu gélosé : } \\
\text { - colonie muqueuse } \\
\text { - pigmentation brun noirâtre }\end{array}$ & $\pm 1-1+$ & $+1-$ \\
\hline 3 Cellules & ovales ou rondes & rondes ou ovales \\
\hline $\begin{array}{l}4 \text { Filamentation : } \\
\text { - absente } \\
\text { - tubes mycéliens } \\
\text { - ébauches de pseudomycélium } \\
\text { - pseudomycélium }\end{array}$ & $\begin{array}{l}+1- \\
-1+ \\
-1+ \\
-1+\end{array}$ & $\begin{array}{l}+1- \\
-1+ \\
-1+\end{array}$ \\
\hline
\end{tabular}


TABleaue III. - (Suite)

\begin{tabular}{|c|c|c|}
\hline & v. albidus & v. diffluens \\
\hline 5 Fermentation & absente & absente \\
\hline $\begin{array}{l}6 \text { Assimilation : } \\
\text { - Glucose } \\
\text { - Galactose } \\
\text { - L Sorbose } \\
\text { - Saccharose } \\
\text { - Maltose } \\
\text { - Cellobiose } \\
\text { - Tréhalose } \\
\text { - Lactose } \\
\text { - Mélibiose } \\
\text { - Raffinose } \\
\text { - Mélézitose } \\
\text { - Inuline } \\
\text { - D Xylose } \\
\text { - L Arabinose } \\
\text { - D Ribose } \\
\text { - L Rhamnose } \\
\text { - Alcool } \\
\text { - Erythritol } \\
\text { - Adonitol } \\
\text { - Dulcitol } \\
\text { - D Mannitol } \\
\text { - D Sorbitol } \\
\text { - Alpha méthyl-D-glucoside } \\
\text { - Inositol } \\
\text { - Nitrate de potassium }\end{array}$ & $\begin{array}{l}+ \\
+1+\mathrm{f} \\
+1+\mathrm{f} /- \\
+ \\
+ \\
+1+\mathrm{f} \\
+1+\mathrm{f} \\
+1+\mathrm{f} \\
-1+\mathrm{f} \\
+1+\mathrm{f} /- \\
+1+\mathrm{f} \\
- \\
+1+\mathrm{f} \\
+1+\mathrm{f} /- \\
+1+\mathrm{f} /- \\
+1+\mathrm{f} /- \\
+1+\mathrm{f} /- \\
+1+\mathrm{f} /- \\
+1+\mathrm{f} \\
+1+\mathrm{f} \\
+1+\mathrm{f} /- \\
+1+\mathrm{f} \\
+1+\mathrm{f}\end{array}$ & $\begin{array}{l} \\
-1+\mathrm{f} /+ \\
-1+\mathrm{f} \\
+1+\mathrm{f} \\
+1+\mathrm{f} \\
+1+\mathrm{f} \\
+1+\mathrm{f} \\
- \\
+1+\mathrm{f} /- \\
+1+\mathrm{f} \\
+1+\mathrm{f} \\
+1+\mathrm{f} \\
-1+\mathrm{f} \\
-1+\mathrm{f} /+ \\
+1+\mathrm{f} /- \\
-1+\mathrm{f} \\
-1+\mathrm{f} /+ \\
-1+\mathrm{f} \\
+1+\mathrm{f} /- \\
+1+\mathrm{f} \\
+\mathrm{f} /+1- \\
+1+\mathrm{f} \\
+\end{array}$ \\
\hline 7 Température maximale & $30^{\circ}-37^{\circ}$ & $32^{\circ}-38^{\circ}$ \\
\hline $\begin{array}{l}8 \text { Sur milieu actidione à } 0,5 \mathrm{~g} / \mathrm{L} \text { : } \\
\text { - développement }\end{array}$ & - & - \\
\hline $\begin{array}{l}9 \text { Sur milieu tétrazolium : } \\
\text { - à } 1 / 1000 \text {, développement }\end{array}$ & $+1-$ & $-1+$ \\
\hline
\end{tabular}

$+\mathrm{f}=$ réaction faiblement positive

3 - La séparation en deux espèces, $C$, albidus et $C$. diffluens (4), est moins fondée depuis les approches nouvelles de ces microorganismes. La réunion des deux entités en une seule est défendable, eu égard aux levures décrites avec un grand nombre de caractères variables. Néanmoins, la distinction en deux variétés, option de Phaff et Fell (5), nous semble, pour lors, préférable.

Une polyphagie et une résistance au tétrazolium plus grandes chez albidus, une tendance à l'arrondissement des blastospores et une thermotolérence plus élevée chez diffluens, distinguent généralement les deux variétés. Chez ces deux Cryptococcus, qui ont probablement la même origine, dont le profil assimilitif demeure encore assez instable, il existe une différenciation morphologique nuancée et une divergence physiologique plus nette. 


\section{BIBLIOGRAPHIE}

I. Dabbagh R., Conant N. F., Burns R. O. : Effect of temperature on saprophytic Cryptococci : observation relatine to wall biosynthesis at non-permissive growth temperatures. $J$. Gen. Microbiol., 1974, 85, 190-202.

2. Dabbagh R., Conant N. F., Nielsen H. S., Burns R. C. : Effect of temperature on saprophytic Cryptococci : temperature-induced lysis and protoplast formation. J. Gen. Microbiol., 1974, 85, 177-189.

3. Kreger-van rij N. J. W. : The genus Cryptococcus. Ann. Soc. Belge Méd. Trop., I964, 44, 60I-6Io.

4. Lodder J., Kreger-van riJ N. J. W. : The yeasts. A taxonomic study. I vol. North-Holland Publ. Cy., Amsterdam, I952.

5. Phaff H. J., Fell J. W. : The genus Cryptococcus. In : Lodder J. Ed. The yeasts. A taxonomic study. I vol., pp. I088-I 145. North-Holland Publ. Cy., Amsterdam-London, I970.

6. SAez H. : Étude de 29 souches de Cryptococcus isolées en cinq ans chez des Mammifères et des Oiseaux. Rev. Mycol., 1965, 30, 57-73.

7. SAEz H. : Réservoir animal, caractéristiques et différenciation de trois Cryptococcus voisins : C. neojormans, C. uniguttulatus et C. luteolus. Zbl. Vet. Med., B, 1978, 25, 764-774.

8. Uden N. VAn, Do CARmo Sousa L. : Yeasts from the bovine caecum. J. Gen. Microbiol., I957, I6, 385-395.

9. Widra A., Long I. : Taxonomic studies on Cryptococci and related yeasts. Mycopath. Mycol. appl., 1970, 40, 89-96. 